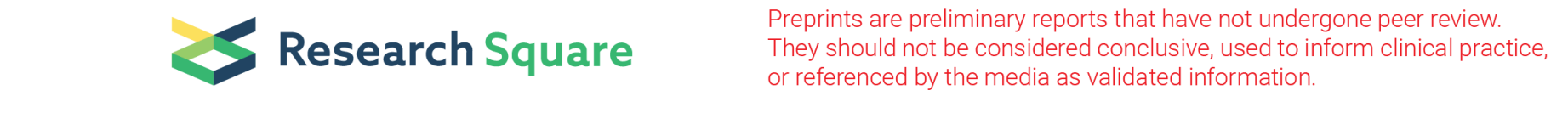

\title{
Do Winter Birds Prefer Urban Forest Fragments Or Residential Areas?
}

\author{
Ryan Buron ( $\sim$ reburon32@gmail.com ) \\ University of Florida https://orcid.org/0000-0003-0371-0449 \\ Mark Hostetler \\ University of Florida \\ Michael Andreu \\ University of Florida
}

\section{Research Article}

Keywords: Urban ecology, winter habitat, birds, forest fragments

Posted Date: January 12th, 2022

DOI: https://doi.org/10.21203/rs.3.rs-1043805/v1

License: () (i) This work is licensed under a Creative Commons Attribution 4.0 International License. Read Full License 


\section{Abstract}

Although urban habitats might not be suitable breeding habitat for many bird species, they can provide valuable wintering habitat. Specifically, residential areas and urban forest fragments can be used by birds during the non-breeding times. Study objectives were: (1) to determine which species primarily use urban forest fragments versus adjacent residential areas during winter; and (2) to assess the influence of species functional traits on habitat preference. Conducted in Gainesville, Florida, 36 random points were situated in urban forest fragments, adjacent residential areas, and forest-residential edges during the winter of 2020. Forty-one total species were observed throughout the study area. Thirteen species (32\%) significantly preferred residential areas, 6 (15\%) significantly preferred urban forest fragments, and 22 (53\%) showed no significant preference to a habitat. Of the birds that significantly preferred residential habitats, there were 4 frugivores species (100\%), 2 omnivore species (29\%), and 4 granivore species (57\%). Five (23\%) insectivore species preferred fragments, but $3(13 \%)$ preferred the residential habitat. Analyses demonstrated that forest fragments contained more vertical vegetation structure with more vegetation at the ground, understory, and canopy levels. Residential habitats and urban forest fragments were able to support a wide variety of bird species during the winter in Gainesville. These results point towards the importance of conserving vegetation in residential habitats, including large trees and understory vegetation, as well as retaining small forest fragments throughout urban areas as habitat for birds during the winter.

\section{Introduction}

Urbanization is one of the main threats to wildlife and the most significant cause of species becoming endangered due to habitat loss (Czech et al. 2000; Sushinsky et al. 2013). In forested areas, habitat is lost in the conversion from forest to urban; however, cities often retain remnant forest fragments and conserve trees within residential areas. Both forest fragments and residential vegetation cover can provide habitat for many species, increasing biodiversity across the urban matrix (Braaker et al. 2014). In particular, these areas can provide refuge from predators, food resources, and connectivity in the landscape for many bird species (Hostetler \& Holling 2000; Dawson \& Hostetler 2010; Lepczyk \& Warren 2012; Marzluff 2017; Archer et al. 2019). For example, avian species richness in urban habitats has been positively associated with forest connectivity in urban landscapes (Evans et al. 2009; Shanahan et al. 2011; Kang, Minor, Park, \& Lee 2015). Even individual trees and small patches of trees may be important for avian movements in urban landscapes (Threlfall et al. 2012). As urban environments continue to grow, it is important to understand how birds respond to the different habitats in cities.

To understand why some species use specific urban habitats, it is important to identify which ecological and life history traits of birds are associated with these urban habitat types (Bonier et al. 2007; Møller 2009; McDonnell \& Hahs 2015). Identifying these traits can improve our understanding of avian habitat preferences, and facilitate species conservation through habitat preservation, management, and restoration (Callaghan et al. 2019). Previous studies have found that avian ecological traits vary in urban environments, including diet (Fuller et al. 2008; Evans et al. 2011; Beissinger \& Osborne 2019); range size (Huhta 2000; Chace \& Walsh 2006; Croci et al. 2008); and migratory status (Friesen et al. 1995). Breeding season studies have shown that urbanized landscapes typically favor omnivores, ground foragers, and seed eaters, while other species such as foliage foragers and insectivores, struggle in urban areas (Chace \& Walsh 2006). However, increasing vegetative structure and cover in urban areas may mitigate the negative impacts of urbanization (Evans et al. 2009; Hostetler 2012).

Many urban bird studies include just the breeding season, but the ecology of winter bird communities and factors that are important for winter bird conservation are not well known (Jadczyk \& Drzeniecka-Osiadacz 2013; Jokimäki \& Kaisanlahti-Jokimäki 2012; Faaborq et al. 2010)). A few studies during the winter show winter habitat needs vary from breeding requirements (Faaborq et al. 2010) and that heavily vegetated urban areas can support diverse winter bird communities (Jokimäki \& Suhonen 1998; Hostetler \& Holling 2000). A systematic review by Amaya-Espinel and Hostetler (2019) demonstrated that many birds species that typically breed in large forest tracts in North America are found in urban forest fragments and residential areas during migration and winter seasons in South America. Also, Spurr (2012) found that the presence of exotic vegetation in residential yards provides novel food resources for birds throughout the year, that are typically not available from native plants. This may be especially important for frugivores and nectivores (Spurr 2012).

Migrating species spend as much as six to seven months in specific wintering habitat (Holmes 2007). Disruption of winter bird habitat can potentially have drastic population implications, regardless of the management of land at the breeding grounds (Holmes 2007). To investigate whether certain avian species prefer different urban habitats with different vegetation structure during the 
winter, we designed a study to measure avian use of forest fragments versus residential areas in Gainesville, Florida. Gainesville includes a mixture of urban forest remnants that are situated nearby to residential areas that typically contain greater than $50 \%$ of tree canopy coverage. Gainesville has been designated a Tree City USA by the Arbor Foundation and has policies and strategies to retain tree canopy cover across the city. Also, Gainesville supports numerous overwintering migrant species as well as resident species (Rowan \& Manetz 2006). This diversity provides a range of dietary guilds to explore whether diet influences which habitats birds utilized in the winter.

The objectives of our study were 1) to determine which bird species were using urban forest fragments versus adjacent residential areas during the winter season, and 2) to assess the influence of species functional traits on habitat preference. Because forest fragments have more vertical height structure, which provides more foraging opportunities under the canopy (Evans et al., 2009), we predicted that understory insectivores would use the urban forest fragments more than residential areas. We also predicted that granivores and frugivores will be more likely to use residential areas because of increased foraging opportunities due to typically more exotic and native fruiting trees (Gray \& van Heezik 2016) and supplementary seed feeders put out by humans in residential areas (Galbraith et al. 2015).

\section{Methods}

\subsection{Study Area and Sampling Design}

This study was conducted in Gainesville, Florida during the winter of 2020. Gainesville is an inland city in northcentral Florida with a humid subtropical climate. It has a population of 132,127 with a density of 2,140 per square mile (US Census Bureau 2019). Twentynine percent of Gainesville is classified as residential habitat and 27 percent is classified as forest (Andreu et al. 2017). The average canopy cover throughout Gainesville is 47 percent, which is higher than other larger cities in Florida, like Orlando, Miami, and Tampa Bay (Andreu et al. 2017). Loblolly pine (Pinus taeda), slash pine (Pinus elliottii), laurel oak (Quercus laurifolia), and water oak (Quercus nigra) are the most common species observed throughout the city of Gainesville (Andreu et al. 2017).

In our study, we included 5 forest fragments and adjacent residential areas throughout Gainesville, Florida: Loblolly Woods Nature

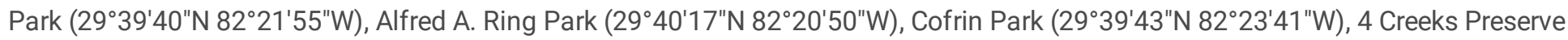
$\left(29^{\circ} 43^{\prime} 9^{\prime \prime} \mathrm{N} 82^{\circ} 22^{\prime} 31^{\prime \prime W}\right)$ and Devils Millhopper $\left(29^{\circ} 42^{\prime} 21^{\prime \prime} \mathrm{N} 82^{\circ} 23^{\prime} 41^{\prime \prime W}\right)$. All forest fragments, which were between 20 acres and 400 acres, had adjacent residential areas with canopy cover. These urban forest fragments are used for jogging, walking, and other nonconsumptive recreational activities. We selected sites that were in the urban sprawl of the city.

We censused birds at 36 point-count locations in urban forest fragments, adjacent residential areas, and the edges between the forest fragments and residential areas in Gainesville, Florida. All point counts had a 50-meter radius and locations were randomly located in the following manner. One surveyor competed all surveys (RB). At each urban forest fragment, we generated random points on the perimeter of the fragment using ArcMap random point generator. At each random point, we then created 3 matched survey points located 1) on the edge of the fragment and adjacent neighborhood, 2) a random distance between $150 \mathrm{~m}$ and $350 \mathrm{~m}$ inside of the fragment, and 3) the same random distance inside of the residential area (Figure 1). Each survey location was at least 150 meters apart to establish independence (Petit et al. 1995; Krementz et al. 1997).

\subsection{Bird Surveys}

We conducted avian point counts from 29 December 2019 to 20 March 2020. We surveyed each site 13 times throughout the season. At each survey location, we completed 10-minute point counts with repeated surveys separated by at least 4 days (Sutherland et al. 2004). We recorded all the birds observed or heard during the survey period. We sampled each set of 3 matched survey points synchronously. To account for variation in daily fluctuation of species and weather, we surveyed all points in a forest fragment and adjacent residential area in the same day. To adjust for sampling bias due to time of day, we systematically rotated start times for each survey (Buler et al. 2007). We conducted the point-count surveys beginning at sunrise and we stopped 4 hours after sunrise. To maximize detection of birds, we did not survey on days with wind averaging over $15 \mathrm{mph}$ or excessive rain.

\subsection{Vegetation Surveys}


At the center of each point-count location, we conducted rapid vegetation surveys to compare the canopy, understory, and ground vegetation between residential habitats and urban forest fragments. At each site, we created 2 random 30-degree wedges from the center of the survey location to the edge of the boundary $(50 \mathrm{~m})$. In each wedge, we counted the number of the trees above $6 \mathrm{~cm} \mathrm{DBH}$ and counted the number of trees under $6 \mathrm{~cm} \mathrm{DBH}$. In the entire point count area, we estimated percent of the ground covered by vegetation, impervious surface, bare soil, and mowed grass. We estimated tree canopy cover by using Google Earth imagery. To do this, we first created a 50m radius around each survey point in Google Earth. We then overlaid a grid in the survey area and counted the number of intersecting points of the grid that had canopy cover. This was then converted into a percent canopy cover for each survey point (Landry et al. 2021). To compare overall differences in vegetation between residential and forest fragment areas, we used a MANOVA and then univariate F-tests to test differences in the individual vegetative measures between habitats.

\subsection{Statistical Analysis}

All statistical analysis were carried out in the R 3.5.0 (R Core Team 2020) statistical environment using function provided with the package's stats and vegan (Oksanen 2011; R Core Team 2020). We included both wintering migrants and year-around resident species in our analysis. We excluded from our analyses any birds that we were unable to identify to species (i.e., woodpecker species drumming).

\subsubsection{Individual Species}

To understand the preference of individual species to forest fragments or residential areas, we used a G-test of goodness-of-fit (Sokal $\&$ Rohlf 2012) for species that had 10 or more total observations (alpha $=0.05$ ). If the number of total observations was less than 10 , we used an exact test of goodness-of-fit (Bower 2003). For individual species analysis, we separated the edge observations into either fragment or residential observations. During the edge surveys, we documented whether each bird was first observed in the fragment or residential area. If the bird was first observed in the forest fragment, then the observation was added to the fragment occurrences. If the bird was first observed in the residential area, then the observation was added to the residential occurrences.

\subsubsection{Functional Trait Response}

We also looked at the functional guild-level response of birds between forest fragments and residential areas. We focused on foraging guilds (nectar and fruit eaters, granivores, omnivores, and insectivores), migratory guilds (winter migrants and resident species), and vertical strata (under canopy and canopy). To group species in dietary and vertical strata guilds, we used the Elton trait database (Wilman et al. 2014). We classified species that spent at least 60 percent of their time in the canopy or mid-canopy categories in the Elton trait database as canopy birds, and species that spent less than 60 percent of their time in the canopy we classified as under-canopy birds. We determined dietary groups from the classification from the Elton trait database (Wilman et al. 2014). We determined migratory status using expert opinion, eBird records for Gainesville, and Cornell's All about Birds range maps (Cornell Lab of Ornithology 2019). We used the results of the individual species preferences to compare function guild-level responses of birds between fragments and residential areas. We reported the proportions of species that significantly preferred residential or fragment habitats in each of the functional guild categories.

\section{Results}

We observed a total of 41 winter migrants and resident species in Gainesville during the winter survey period. All 41 species were observed at more than one survey location. A complete list of all the birds observed during this study can be found in Table 1. 
Table 1

List of bird species observed during winter surveys in Gainesville, Florida. The summed abundance of each species at each habitat, the g-score, $P$-value, direction of significance (if applicable), and guild categorizations are listed.

\begin{tabular}{|c|c|c|c|c|c|c|c|c|c|}
\hline $\begin{array}{l}\text { Common } \\
\text { name }\end{array}$ & $\begin{array}{l}\text { Scientific } \\
\text { name }\end{array}$ & Fragment & Residential & $\begin{array}{l}\text { g- } \\
\text { score }\end{array}$ & $\begin{array}{l}P \\
\text { value }\end{array}$ & direction & $\begin{array}{l}\text { Vertical } \\
\text { Strata }\end{array}$ & Foraging & $\begin{array}{l}\text { Migratory } \\
\text { Status }\end{array}$ \\
\hline $\begin{array}{l}\text { Baltimore } \\
\text { Oriole }\end{array}$ & $\begin{array}{l}\text { lcterus } \\
\text { galbula }\end{array}$ & 0 & 26 & 36.04 & $<0.001$ & Res & Canopy & Fruit & Winter \\
\hline $\begin{array}{l}\text { Cedar } \\
\text { Waxwing }\end{array}$ & $\begin{array}{l}\text { Bombycilla } \\
\text { cedrorum }\end{array}$ & 11 & 224 & 236.95 & $<0.001$ & Res & Canopy & Fruit & Winter \\
\hline $\begin{array}{l}\text { Blue-headed } \\
\text { Vireo }\end{array}$ & $\begin{array}{l}\text { Vireo } \\
\text { solitarius }\end{array}$ & 9 & 2 & 4.82 & 0.028 & Frag & Canopy & Invert & Winter \\
\hline $\begin{array}{l}\text { Orange- } \\
\text { crowned } \\
\text { Warbler }\end{array}$ & $\begin{array}{l}\text { Vermivora } \\
\text { celata }\end{array}$ & 5 & 3 & NA & 0.727 & - & Canopy & Invert & Winter \\
\hline $\begin{array}{l}\text { Yellow- } \\
\text { throated } \\
\text { Warbler }\end{array}$ & $\begin{array}{l}\text { Setophaga } \\
\text { dominica }\end{array}$ & 10 & 5 & 1.70 & 0.192 & - & Canopy & Invert & Winter \\
\hline $\begin{array}{l}\text { Downy } \\
\text { Woodpecker }\end{array}$ & $\begin{array}{l}\text { Picoides } \\
\text { pubescens }\end{array}$ & 45 & 49 & 0.17 & 0.680 & - & Canopy & Invert & Year \\
\hline $\begin{array}{l}\text { Pileated } \\
\text { Woodpecker }\end{array}$ & $\begin{array}{l}\text { Dryocopus } \\
\text { pileatus }\end{array}$ & 36 & 26 & 1.62 & 0.203 & - & Canopy & Invert & Year \\
\hline Pine Warbler & $\begin{array}{l}\text { Setophaga } \\
\text { pinus }\end{array}$ & 8 & 16 & 2.72 & 0.099 & - & Canopy & Invert & Year \\
\hline $\begin{array}{l}\text { Tufted } \\
\text { Titmouse }\end{array}$ & $\begin{array}{l}\text { Baeolophus } \\
\text { bicolor }\end{array}$ & 50 & 38 & 1.64 & 0.200 & - & Canopy & Invert & Year \\
\hline $\begin{array}{l}\text { Yellow-bellied } \\
\text { Sapsucker }\end{array}$ & $\begin{array}{l}\text { Sphyrapicus } \\
\text { varius }\end{array}$ & 6 & 2 & NA & 0.289 & - & Canopy & Omni & Winter \\
\hline $\begin{array}{l}\text { Reb-bellied } \\
\text { Woodpecker }\end{array}$ & $\begin{array}{l}\text { Melanerpes } \\
\text { carolinus }\end{array}$ & 41 & 40 & 0.01 & 0.912 & - & Canopy & Omni & Year \\
\hline House Finch & $\begin{array}{l}\text { Haemorhous } \\
\text { mexicanus }\end{array}$ & 4 & 7 & 0.82 & 0.363 & - & Canopy & Seed & Year \\
\hline $\begin{array}{l}\text { American } \\
\text { Robin }\end{array}$ & $\begin{array}{l}\text { Turdus } \\
\text { migratorius }\end{array}$ & 1 & 25 & 27.57 & $<0.001$ & Res & Under & Fruit & Winter \\
\hline $\begin{array}{l}\text { Northern } \\
\text { Mockingbird }\end{array}$ & $\begin{array}{l}\text { Mimus } \\
\text { polyglottos }\end{array}$ & 1 & 31 & 35.46 & $<0.001$ & Res & Under & Fruit & Year \\
\hline $\begin{array}{l}\text { Black-and- } \\
\text { white Warbler }\end{array}$ & Mniotilta varia & 14 & 9 & 1.10 & 0.295 & - & Under & Invert & Winter \\
\hline $\begin{array}{l}\text { Blue-gray } \\
\text { Gnatcatcher }\end{array}$ & $\begin{array}{l}\text { Polioptila } \\
\text { caerulea }\end{array}$ & 20 & 11 & 2.65 & 0.103 & - & Under & Invert & Winter \\
\hline
\end{tabular}


Table 1

Continued

\begin{tabular}{|c|c|c|c|c|c|c|c|c|c|}
\hline $\begin{array}{l}\text { Common } \\
\text { name }\end{array}$ & Scientific name & Fragment & Residential & $\begin{array}{l}\text { g- } \\
\text { score }\end{array}$ & $\begin{array}{l}\mathrm{p}- \\
\text { value }\end{array}$ & direction & $\begin{array}{l}\text { Vertical } \\
\text { Strata }\end{array}$ & Foraging & $\begin{array}{l}\text { Migratory } \\
\text { Status }\end{array}$ \\
\hline $\begin{array}{l}\text { Eastern } \\
\text { Phoebe }\end{array}$ & Sayornis phoebe & 9 & 13 & 0.73 & 0.393 & - & Under & Invert & Winter \\
\hline Gray Catbird & $\begin{array}{l}\text { Dumetella } \\
\text { carolinensis }\end{array}$ & 56 & 42 & 2.01 & 0.157 & - & Under & Invert & Winter \\
\hline $\begin{array}{l}\text { Hermit } \\
\text { Thrush }\end{array}$ & $\begin{array}{l}\text { Catharus } \\
\text { guttatus }\end{array}$ & 20 & 3 & 14.07 & $<0.001$ & Frag & Under & Invert & Winter \\
\hline Ovenbird & $\begin{array}{l}\text { Seiurus } \\
\text { aurocapilla }\end{array}$ & 9 & 0 & NA & 0.004 & Frag & Under & Invert & Winter \\
\hline $\begin{array}{l}\text { Palm } \\
\text { Warbler }\end{array}$ & $\begin{array}{l}\text { Setophaga } \\
\text { palmarum }\end{array}$ & 0 & 17 & 23.57 & $<0.001$ & Res & Under & Invert & Winter \\
\hline $\begin{array}{l}\text { Prairie } \\
\text { Warbler }\end{array}$ & $\begin{array}{l}\text { Setophaga } \\
\text { discolor }\end{array}$ & 2 & 6 & NA & 0.289 & - & Under & Invert & Winter \\
\hline $\begin{array}{l}\text { Ruby- } \\
\text { crowned } \\
\text { Kinglet }\end{array}$ & $\begin{array}{l}\text { Regulus } \\
\text { calendula }\end{array}$ & 64 & 49 & 2.00 & 0.158 & - & Under & Invert & Winter \\
\hline $\begin{array}{l}\text { Yellow- } \\
\text { rumped } \\
\text { Warbler }\end{array}$ & $\begin{array}{l}\text { Setophaga } \\
\text { coronata }\end{array}$ & 47 & 72 & 5.29 & 0.021 & Res & Under & Invert & Winter \\
\hline $\begin{array}{l}\text { Carolina } \\
\text { Chickadee }\end{array}$ & $\begin{array}{l}\text { Poecile } \\
\text { carolinensis }\end{array}$ & 15 & 26 & 2.99 & 0.084 & - & Under & Invert & Year \\
\hline $\begin{array}{l}\text { Carolina } \\
\text { Wren }\end{array}$ & $\begin{array}{l}\text { Thryothorus } \\
\text { ludovicianus }\end{array}$ & 57 & 67 & 0.81 & 0.369 & - & Under & Invert & Year \\
\hline $\begin{array}{l}\text { Common } \\
\text { Yellowthroat }\end{array}$ & & 25 & 8 & 9.19 & 0.002 & Frag & Under & Invert & Year \\
\hline $\begin{array}{l}\text { Eastern } \\
\text { Bluebird }\end{array}$ & Sialia sialis & 3 & 55 & 56.79 & $<0.001$ & Res & Under & Invert & Year \\
\hline $\begin{array}{l}\text { Northern } \\
\text { Flicker }\end{array}$ & Colaptes auratus & 9 & 1 & 7.36 & 0.007 & Frag & Under & Invert & Year \\
\hline $\begin{array}{l}\text { White-eyed } \\
\text { Vireo }\end{array}$ & Vireo griseus & 37 & 26 & 1.93 & 0.165 & - & Under & Invert & Year \\
\hline Blue Jay & $\begin{array}{l}\text { Cyanocitta } \\
\text { cristata }\end{array}$ & 13 & 54 & 26.95 & $<0.001$ & Res & Under & Omni & Year \\
\hline $\begin{array}{l}\text { Brown } \\
\text { Thrasher }\end{array}$ & $\begin{array}{l}\text { Toxostoma } \\
\text { rufum }\end{array}$ & 5 & 5 & NA & 1.00 & - & Under & Omni & Year \\
\hline $\begin{array}{l}\text { Common } \\
\text { Grackle }\end{array}$ & $\begin{array}{l}\text { Quiscalus } \\
\text { quiscula }\end{array}$ & 2 & 5 & NA & 0.180 & - & Under & Omni & Year \\
\hline $\begin{array}{l}\text { Eastern } \\
\text { Towhee }\end{array}$ & $\begin{array}{l}\text { Pipilo } \\
\text { erythrophthalmus }\end{array}$ & 15 & 0 & 20.79 & $<0.001$ & Frag & Under & Omni & Year \\
\hline $\begin{array}{l}\text { Red-winged } \\
\text { Blackbird }\end{array}$ & $\begin{array}{l}\text { Agelaius } \\
\text { phoeniceus }\end{array}$ & 12 & 36 & 12.56 & $<0.001$ & Res & Under & Omni & Year \\
\hline
\end{tabular}

Table 1. Continued 


\begin{tabular}{|c|c|c|c|c|c|c|c|c|c|}
\hline Common name & $\begin{array}{l}\text { Scientific } \\
\text { name }\end{array}$ & Fragment & Residential & $\begin{array}{l}\text { g- } \\
\text { score }\end{array}$ & $\begin{array}{l}\mathrm{p}- \\
\text { value }\end{array}$ & direction & $\begin{array}{l}\text { Vertical } \\
\text { Strata }\end{array}$ & Foraging & $\begin{array}{l}\text { Migratory } \\
\text { Status }\end{array}$ \\
\hline $\begin{array}{l}\text { American } \\
\text { Goldfinch }\end{array}$ & Spinus tristis & 0 & 24 & 33.27 & $<0.001$ & Res & Under & Seed & Winter \\
\hline $\begin{array}{l}\text { Chipping } \\
\text { Sparrow }\end{array}$ & $\begin{array}{l}\text { Spizella } \\
\text { passerina }\end{array}$ & 5 & 84 & 84.88 & $<0.001$ & Res & Under & Seed & Winter \\
\hline $\begin{array}{l}\text { Painted } \\
\text { Bunting }\end{array}$ & $\begin{array}{l}\text { Passerina } \\
\text { ciris }\end{array}$ & 0 & 3 & NA & 0.250 & - & Under & Seed & Winter \\
\hline $\begin{array}{l}\text { Brown-headed } \\
\text { Cowbird }\end{array}$ & $\begin{array}{l}\text { Molothrus } \\
\text { ater }\end{array}$ & 0 & 26 & 36.04 & $<0.001$ & Res & Under & Seed & Year \\
\hline $\begin{array}{l}\text { Northern } \\
\text { Cardinal }\end{array}$ & $\begin{array}{l}\text { Cardinalis } \\
\text { cardinalis }\end{array}$ & 75 & 228 & 80.93 & $<0.001$ & Res & Under & Seed & Year \\
\hline Wild Turkey & $\begin{array}{l}\text { Meleagris } \\
\text { gallopavo }\end{array}$ & 10 & 19 & 2.84 & 0.092 & - & Under & Seed & Year \\
\hline
\end{tabular}

\subsection{Individual Species}

We analyzed 34 of the 41 species with a G-test because they had 10 or more observations. The other 7 species were analyzed with an exact test of goodness-of-fit. Thirteen significantly preferred residential areas, 6 significantly preferred urban forest fragments, and 22 showed no significant preference to a habitat (Table 1).

Species that showed a significant preference for forest fragments included: Blue-headed Vireos (Vireo solitarius), Hermit Thrushes (Catharus guttatus), Ovenbirds (Seiurus aurocapilla), Eastern Towhees (Pipilo erythrophthalmus), Common Yellowthroats (Geothlypis trichas) and Northern Flickers (Colaptes auratus). Species that preferred residential habitat included: Baltimore Orioles (Icterus galbula), Cedar Waxwings (Bombycilla cedrorum), Chipping Sparrows (Spizella passerine), American Goldfinches (Spinus tristis), Northern Mockingbirds (Mimus polyglottos), Palm Warblers (Setophaga palmarum), Yellow-rumped Warblers (Setophaga coronate), Eastern Bluebirds (Sialia sialis), Red-winged Blackbirds (Agelaius phoeniceus), American Robins (Turdus migratorius), Northern Cardinals (Cardinalis cardinalis), and Blue Jays (Cyanocitta cristata) (Table 1).

\subsection{Functional Traits}

To categorize bird species into functional trait guilds, we used the following categories: migratory (winter or resident); dietary (frugivore, omnivore, insectivore, or granivore); and vertical strata (canopy or below-canopy). We classified 20 species as winter migrants (only spent the non-breeding season in Gainesville), with 7 (35\%) of the species occurring significantly more often in residential areas, 3 (15\%) significantly more often in fragments, and $10(50 \%)$ did not have a significant preference (Figure 2). We classified the other 21 species as year-round resident species of Gainesville, Florida. Of the year-resident species, $6(28.6 \%)$ of these birds significantly preferred residential habitats, 3 (14.3\%) significantly preferred fragments, and 12 (57.1\%) didn't have any preference (Figure 2). We classified 12 species as canopy affiliated species, with 2 (16.7\%) significantly preferring residential habitats, 1 (8.33\%) preferring urban forest fragments, and 9 (75\%) didn't have a significance preference (Figure 3). We classified the other 29 species as under-canopy foraging species, 11 (37.9\%) of these significantly preferred residential habitats, 5 (17.2\%) preferred forest fragments, and 13 (44.8\%) didn't show a significance preference (Figure 3).

In the dietary guilds, we classified 4 species as frugivores. All 4 of those species, Baltimore Oriole, Cedar Waxwing, American Robin, and Northern Mockingbird, significantly preferred the residential habitat. We classified 23 species as mainly invertebrate foragers, with $3(13 \%)$ preferring residential habitat, 5 (22.8\%) preferring forest fragments, and 15 (65.2\%) didn't show a significant preference (Figure 4). We classified 7 species as omnivores. Two (28.6\%) of these species significantly preferred residential habitats, 1 (14.3\%) significantly preferred forest fragments, and 4 (57.1\%) didn't show a significant preference (Figure 4). Finally, we classified 7 species as granivores with 4 (57.1\%) preferring residential areas, none preferred fragments, and 3 (42.9\%) didn't show a significant preference (Figure 2).

\subsection{Vegetation}


Vegetation characteristics from fragment, residential, and edge habitats differed significantly, as shown in results of the MANOVA ( $P$ $<0.001)$. Univariate F-tests of each vegetative measure showed that percent impervious surface, percent bare soil, percent ground vegetation, percent mowed lawn, small trees, large trees, and canopy cover were all significantly different at the habitat types (Table 2). Canopy heights showed no significant difference among the three habitat types (Table 2). Forest fragments had significantly more trees under $6 \mathrm{~cm} \mathrm{DBH} \mathrm{(F-value}=32.79, P<0.001)$, canopy cover (F-value $=7.505, P=0.0019)$, percent bare soil $(\mathrm{F}$-value $=28.85$, $P<0.001$ ), and percent ground vegetation ( $F$-value $=34.82, P<0.001$ ). Residential habitats had significantly more impervious surface $(F-v a l u e=100.55, P<0.001)$ and percent mowed lawn $(F-v a l u e=60.78, P<0.001)$. All means and statistical significance are listed in Table 2.

Table 2

Habitat parameters measured at each survey site in Gainesville, Florida. Mean of each parameter at each habitat type, the f-statistic, and $P$-value are listed.

\begin{tabular}{|llllll|}
\hline Parameter & fragment & residential & edge & f-statistic & $P$-value \\
\hline \% impervious & 0.00 & 42.50 & 15.00 & 100.55 & $* \star *$ \\
\hline \% ground veg & 62.50 & 10.83 & 32.08 & 34.82 & $* \star *$ \\
\hline \% bare soil & 37.50 & 7.08 & 29.58 & 28.85 & $* \star *$ \\
\hline \% lawn coverage & 0.00 & 39.58 & 22.50 & 60.78 & $* \star *$ \\
\hline \# of large trees & 17.17 & 11.50 & 15.42 & 7.42 & $*$ \\
\hline \# of small trees & 21.75 & 5.83 & 11.50 & 32.79 & $* * *$ \\
\hline canopy height $(\mathrm{m})$ & 22.42 & 23.50 & 23.08 & 0.35 & \\
\hline \% canopy cover & 84.75 & 63.83 & 73.67 & 7.51 & $*$ \\
\hline$* * *=P<0.001, * *=P<0.01, *=P<0.05$ & & & \\
\hline
\end{tabular}

Common canopy species observed at our study sites included: slash pine, laurel oak, live oak (Quercus virginiana), loblolly pine (Pinus taeda), sweetgum (Liquidambar styraciflua), red maple (Acer rubrum), swamp chestnut oak (Quercus michauxii), southern magnolia (Magnolia grandiflora), and sugarberry (Celtis laevigata). Understory and mid-story species included seedlings and sapling of the above species, as well as American hornbeam (Carpinus caroliniana), American elm (Ulmus americana), cabbage palm (Sabal palmetto), dwarf palmetto (Sabal minor), Carolina laurel cherry (Prunus caroliniana), elderberry (Sambucus canadensis) and American beautyberry (Callicarpa americana).

\section{Discussion}

Our study showed that many wintering and year-round resident species used residential areas and adjacent urban forest fragments. We found that more species preferred residential habitats to forest fragments in the winter. Out of the 41 total species we observed, 13 significantly preferred residential habitats and only 6 significantly preferred the adjacent urban forest fragments. Typical yearround resident species such as the Northern Cardinal, Chipping Sparrow, and Eastern Bluebird, unsurprisingly, showed preference for residential areas. These are common urban-affiliated bird species (Seewagen et al. 2010). Chipping sparrows and Northern Cardinals are granivores and may have preferred residential areas due to increases in supplementary feeding (i.e. bird feeders) and sparce vegetation in the residential sites. As seen in previous studies (Chace \& Walsh 2006), granivores are common in residential habitat and thrive in human-influenced areas. A more surprising result was that a few understory and canopy insectivores, like the Yellowrumped Warbler, significantly preferred the residential habitat over the forest fragments or did not prefer one habitat over the other, like the Pine Warbler and Ruby-crowned Kinglet. These migrant species were observed foraging in the mid-canopy, sparce understory vegetation, and on the ground in the residential areas.

The amount of tree canopy and other vegetation at our residential sites may have increased the abundance and diversity of resources available to birds, affecting their habitat preferences. Although such habitats typically include more impervious surface and mowed lawns than nearby forested fragments (Threlfall et al. 2012; Threlfall et al. 2016), and that native plants are often replaced by exotics (Threlfall et al. 2016), the residential areas in our study may have enough vegetation to support a variety of 
species. Residential areas that retain some native vegetation and vegetation structure in yards have been shown to provide habitat for many species (Belaire et al. 2014; Archer et al. 2019). Blair (1996) found that in the oak woodlands of California, total avian richness peaked under moderately disturbed conditions (e.g., golf courses and low-density, detached, single-unit homes).

Even though in our study the forest fragments contained more understory vegetation, ground habitat and slightly more canopy cover than residential sites, there may be enough vegetation in residential areas for some species in the winter. MacArthur (1958) showed that Yellow-rumped Warblers had more flexible foraging strategies than other eastern forest warblers. In wintering grounds, Yellowrumped Warbler (called Myrtle Warblers in the MacArthur paper) foraged from beaches to forests and frequenting open ground. They were common under bayberry plants (Myrica pensylvanica) eating insects or feeding on berries (MacArthur 1958). Yellow-rumped Warblers in our study significantly preferred the residential areas to the urban forest fragments, displaying behavioral flexibility first recorded by MacArthur (1958). Although more canopy cover is associated with greater arthropod species richness and abundance (Turrini \& Knop 2015), the 60 percent canopy cover in the residential neighborhoods may be enough habitat to support species that have flexible foraging strategies. Yellow-rumped Warblers may be foraging not only on arthropods, but some berries and even seeds in feeders. A previous study on the coast of California did show that urban winter bird communities commonly included insectivores like the Yellow-rumped Warbler and Ruby-crowned Kinglet (Kalinowski \& Johnson 2010). These insectivores, in the California study, were associated positively with tall vegetation and negatively with road and building structure cover in the urban landscapes. Another unsurprising result was that the Eastern Bluebird preferred the residential areas. This species has been previously reported preferring open habitats, such as those found in residential neighborhoods (Gowaty \& Plissner 2015). Therefore, residential yards have the potential to provide avian habitat for some insectivores during the winter, especially when canopy cover and vegetation structure is present.

However, other migrants and residents in the insectivore category, Hermit Thrushes, Ovenbirds, Northern Flickers, and Blue-headed Vireos, significantly preferred forest fragments. This suggests that urban forest fragments are still important to retain and manage in order to provide winter habitat for some species. Main et al. (2011) found that the bird community, including year-around species and migrants, in South Florida was negatively associated with native hammock forested fragments, but some species did prefer or showed no difference between hammocks and residential areas. In the South Florida study, birds of conservation concern, such as the migrating Prairie Warbler (Setophaga discolor) and the year-round Mangrove Cuckoo (Coccyzus minor), both preferred the native hammock forest fragments (Main, et al. 2011). In our study, Northern Flickers, the only ground foraging woodpecker, significantly preferred forest fragments. Studies have shown that Northern flickers are rare in Norther California urban and residential yards (Merenlender et al. 2009), so urban forest fragments are a valuable refuge for these species during the winter. Additionally, we only observed Ovenbirds, another common ground foraging species, in forest fragments. Previous work (Hostetler et al. 2005) has shown that increasing urban structures impacts the abundance of Ovenbirds and resulted in their extirpation from a previously wooded area in Gainesville, Florida when it was developed into an apartment complex. In their study, Hostetler et al. (2005) reported Ovenbird occurrences declined from 32 to 3 sightings after an apartment complex was built. Although treed neighborhoods in our study were able to support a variety of wintering species in Florida, forest fragments may be vital for some species, like the Ovenbird and Northern Flicker.

With the foraging guilds, 28 percent of omnivores and 57 percent of granivores preferred residential habitat. In winter, supplementary feeding from humans has been reported to meet the energy need of many granivore species (Lancaster \& Rees 1979). Granivores, like the Northern Cardinal and Chipping Sparrow are able to forage on supplementary feeding from humans (i.e., bird feeders) (Jokimäki \& Suhonen 1998). This is one of the most obvious reasons for the observation that omnivorous and granivorous bird species generally prefer urban habitats and why these species preferred the residential neighborhoods to the urban forest fragments in our study. Galbraith et al. (2015) showed that supplementary feeding, from things like bird feeders, restructured urban bird communities. Our results were consistent with other studies in that most granivore species significantly preferred residential habitat and many omnivore species either significantly preferred residential habitat or showed trends in the residential direction.

All 4 frugivore species, Baltimore Orioles, Cedar Waxwings, Northern Mockingbirds, and American Robins, showed a significant preference for residential habitat during the winter. Residential yards that retain some native plant species, but also have exotic plants can provide alternative foraging opportunities for birds, especially frugivores. A study by Andreu et al. (2017) showed that residential areas in Gainesville contained 70 species of trees, while forest areas only contained 42 species. Although the forest areas had more native species, the residential areas contained more species, especially exotics (Andreu et al., 2017). In another urban study, residential habitats in Australia were found to contain higher plant species richness because of the ornamental shrubs and 
exotic plants (Threlfall et al. 2016). Gleditsch and Carlo (2011) also found a positive association between the abundance of some birds and an exotic plant species, honeysuckles (Lonicera spp.), in Pennsylvania. This was not only beneficial for the honeysuckle species, but in areas of high honeysuckle density, native trees had 30\% higher fruit removal rates compared to areas with low density of honeysuckle (Gleditsch \& Carlo 2011). While the exotic fruits might attract the birds, native fruit bearing species also benefitted. Exotic plant species may have provided extra foraging opportunities for frugivores in Gainesville residential areas.

We also found that some winter migrants that were observed in another migration study (Buron, unpublished data) preferred similar habitats during both seasons. Species like the Ovenbird and Eastern Towhee had the same habitat preference during migration and winter. The Eastern Towhee almost exclusively used forest fragments during the winter and migration. This results corresponds with other studies that claim that Eastern Towhees are threatened by urban development (Seewagen et al. 2010), suggesting forest fragments are important stopover and wintering habitat for this species. Further, the Black-and-white Warbler significantly preferred forest fragments during migration (Buron, unpublished data), but in this study, during the winter period, they had no significant preference. Also, the Common Yellowthroat significantly preferred fragments during winter, but in the previous migration study, it had no preference. Further investigation is needed to understand this difference in habitat preference during migration and winter.

Of note, we did not address whether a species would be foraging in a residential area if there were not nearby forest fragments. All our residential surveys were near urban forest fragments; it is possible that a heavily treed residential area that is situated far from forest fragments may not contain certain species during the winter. More comparative studies are needed to ascertain whether larger landscape features affect bird distributions.

\subsection{Recommendations for Urban Planning.}

This study found that a variety of winter birds can use both residential areas with tree canopy and forest fragments in the urban matrix. Even migrating species that are classified as interior forest breeders, like the Black-and-white Warbler (Archer et al. 2019), still used residential trees and urban forest fragments as overwintering habitat in Gainesville. Other under-canopy insectivore species like the Palm Warbler and Yellow-rumped Warbler significantly preferred residential habitat. Another common under-canopy insectivore, the Ruby-crowned Kinglet, used both forest fragments and residential habitats frequently. These results point to the importance of not just looking at patterns in groups or guilds of species, but also studying individual species response. Studies documenting individual species preference can point to results that are missed from just looking at bird community and guild studies. Overall, this study adds to the evidence that vegetation at residential yards and conserving forested habitats in the urban matrix, impact bird diversity and contribute to conservation of vital bird habitat. Although planners and developers often prioritize large forest fragments for conservation, small forest fragments and landscaping in residential yards can help provide winter habitat for migrant and resident bird species.

\section{Declarations}

Funding: This research was in part funded by was supported by the USDA National Institute of Food and Agriculture, Renewable Resources Extension Act, UF/IFAS project 1000606 and by the USDA National Institute of Food and Agriculture Hatch project 1025925

Conflicts of interest/Competing interests: Not applicable

Availability of data and material: Not applicable

Code availability: Not applicable

Authors' contributions: Not applicable

\section{References}

1. Amaya-Espinel, J. D., \& Hostetler, M. E. (2019). The value of small forest fragments and urban tree canopy for Neotropical migrant birds during winter and migration seasons in Latin American countries: A systematic review. Landscape and Urban Planning, 190. https://doi.org/10.1016/j.landurbplan.2019.103592. 
2. Andreu, M. G., Fox, D. A., Landry, S. M., Northrop, R. J., \& Hament, C. A. (2017). Urban Forest Ecological Analysis. Report to the City of Gainesville, March 2017. Gainesville, Florida.

3. Archer, J. M. J., Hostetler, M. E., Acomb, G., \& Blair, R. (2019). A systematic review of forest bird occurrence in North American forest fragments and the built environment. Landscape and Urban Planning, 185, 1-23. https://doi.org/10.1016/j.landurbplan.2019.01.005.

4. Beissinger, S. R., \& Osborne, D. R. (2019). Effects of Urbanization on Avian Community. Condor, 84(1), 75-83.

5. Belaire, J. A., Whelan, C. J., \& Minor, E. S. (2014). Having our yards and sharing them too: The collective effects of yards on native bird species in an urban landscape. Ecological Applications, 24(8), 2132-2143. https://doi.org/10.1890/13-2259.1.

6. Blair, R. B. (1996). Land Use and Avian Species Diversity Along an Urban Gradient. Ecological Society of America, 6(2), 506-519.

7. Bonier, F., Martin, P. R., \& Wingfield, J. C. (2007). Urban birds have broader environmental tolerance. Biology Letters, 3(6), 670673. https://doi.org/10.1098/rsbl.2007.0349.

8. Braaker, S., Moretti, M., Boesch, R., Ghazoul, J., Obrist, M. K., \& Bontadina, F. (2014).

9. Assessing habitat connectivity for ground-dwelling animals in an urban environment. Ecological Applications, 24(7), 15831595. https://doi.org/10.1890/13-1088.1.

10. Buler, J. J., Moore, F. R., \& Woltmann, S. (2007). A multi-scale examination of stopover habitat use by birds. Ecology, 88(7), 1789-1802. https://doi.org/10.1890/06-1871.1.

11. Callaghan, C. T., Major, R. E., Lyons, M. B., Martin, J. M., Wilshire, J. H., Kingsford, R. T., \&

12. Cornwell, W. K. (2019). Using citizen science data to define and track restoration targets in urban areas. Journal of Applied Ecology, (May), 1998-2006. https://doi.org/10.1111/1365-2664.13421.

13. Chace, J. F., \& Walsh, J. J. (2006). Urban effects on native avifauna: A review. Landscape and

14. Urban Planning, 74(1), 46-69. https://doi.org/10.1016/j.landurbplan.2004.08.007.

15. Cornell Lab of Ornithology. 2019. All About Birds. Cornell Lab of Ornithology, Ithaca, New

16. York. https://www.allaboutbirds.org. Accessed on 5 May 2020.

17. Croci, S., Butet, A., \& Clergeau, P. (2008). Does Urbanization Filter Birds on the Basis of Their

18. Biological Traits? The Condor, 110(2), 223-240. https://doi.org/10.1525/cond.2008.8409.

19. Czech, B., Krausman, P. R., \& Devers, P. K. (2000). Economic associations among causes of species endangerment in the United States. BioScience, 50(7), 593-601. https://doi.org/10.1641/0006-3568(2000)050[0593:EAACOS]2.0.C0;2.

20. Dawson, D., \& Hostetler, M. (2010). Edge Avoidance by Birds Within Urban Forest Remanents. Florida Scientist, 73(3/4), 203217.

21. Escobedo, F., Seitz, J. A., \& Zipperer, W. (2009). Gainesville's Urban Forest Canopy Cover. In UF IFAS extension.

22. Evans, K. L., Chamberlain, D. E., Hatchwell, B. J., Gregory, R. D., \& Gaston, K. J. (2011). What makes an urban bird? Global Change Biology, 17(1), 32-44. https://doi.org/10.1111/j.1365-2486.2010.02247.x.

23. Evans, K. L., Newson, S. E., \& Gaston, K. J. (2009). Habitat influences on urban avian assemblages. Ibis, 151(1), 19-39. https://doi.org/10.1111/j.1474-919X.2008.00898.x.

24. Faaborq, J., Holmes, R. T., Anders, A. D., Bildstein, K. L., Dugger, K. M, Gauthreaux, S. A.,

25. Heglund, P., Hobson, K. A., Jahn, A. E., Johnson, D. H., Latta, S. C., Levey, D. J., Marra, P. P., Merkord, C. L., Nol, E., Rothstein, S. I., Sherry, T.W., Sillett, T. S., Thompson, F. R., \& Warnock, N. 2010. Recent advances in understanding migration systems of New World land birds. Ecological Monographs 80(1): 3-48. https://doi.org/10.1890/09-0395.1.

26. Friesen, L. E., Eagles, P. F. J., \& Mackay, R. J. (1995). Effects of Residential Development on Forest-Dwelling Neotropical Migrant Songbirds. Conservation Biology, 9(6), 1408-1414. https://doi.org/10.1046/j.1523-1739.1995.09061408.x.

27. Fuller, R. A., Warren, P. H., Armsworth, P. R., Barbosa, O., \& Gaston, K. J. (2008). Garden bird

28. feeding predicts the structure of urban avian assemblages. Diversity and Distributions, 14(1), 131-137. https://doi.org/10.1111/j.1472-4642.2007.00439.x.

29. Gowaty, Patricia A. \& Plissner, Jonathan A. (2015). Eastern Bluebird (Sialia sialis), version 2.0. In The Birds of North America (P. G. Rodewald, editor). Cornell Lab of Ornithology, Ithaca, New York, USA.

30. Holmes, R. T. (2007). Understanding population change in migratory songbirds: Long-term and 
31. experimental studies of Neotropical migrants in breeding and wintering areas. Ibis, 149(SUPPL. 2), 2-13. https://doi.org/10.1111/j.1474-919X.2007.00685.x.

32. Hostetler, M. 2012. The Green Leap: A Primer for Conserving Biodiversity in Subdivision Development. University of California Press, Berkeley, CA. 185 pp

33. Hostetler, M., Duncan, S., \& Paul, J. (2005). Post-construction Effects of an Urban Development on Migrating, Resident, and Wintering Birds. Southeastern Naturalist, 4(3), 421-434. https://doi.org/10.1656/1528-7092(2005)004[0421:peoaud]2.0.co;2.

34. Hostetler, M., \& Holling, C. S. (2000). Detecting the scales at which birds respond to structure in urban landscapes. Urban Ecosystems, 4, 25-54. https://doi.org/10.1023/A:1009587719462.

35. Jadczyk, P., \& Drzeniecka-Osiadacz, A. (2013). Feeding strategy of wintering rooks Corvus frugilegus L. in urban habitats. Polish Journal of Ecology, 61(3), 587-596.

36. Jokimaki, J., \& Huhta, E. (2000). Artificial Nest Predation and Abundance of Birds along an Urban Gradient. The Condor, 102, 838-847. https://doi.org/10.2307/1370311.

37. Jokimäki, J., \& Kaisanlahti-Jokimäki, M.-L. (2012). The role of residential habitat type on the temporal variation of wintering bird assemblages in northern Finland. Ornis Fennica, 89(1), 20-33.

38. Jokimäki, J., \& Suhonen, J. (1998). Distribution and habitat selection of wintering birds in urban environments. Landscape and Urban Planning, 39, 253-263. https://doi.org/10.1016/S0169-2046(97)00089-3.

39. Kalinowski, R. S., \& Johnson, M. D. (2010). Influence Of Suburban Habitat on a Wintering Bird Community in Coastal Northern California. The Condor, 112(2), 274-282. https://doi.org/10.1525/cond.2010.090037.

40. Kang, W., Minor, E. S., Park, C. R., \& Lee, D. (2015). Effects of habitat structure, human disturbance, and habitat connectivity on urban forest bird communities. Urban Ecosystems, 18(3), 857-870. https://doi.org/10.1007/s11252-014-0433-5.

41. Krementz, D. G., Ralph, C. J., Sauer, J. R., \& Droege, S. (1995). Monitoring Bird Populations by

42. Point Counts. In USDA Forest Service. https://doi.org/10.2307/3802161.

43. Lancaster, R. K., \& Rees, W. E. (1979). Bird communities and the structure of urban habitats.

44. Canadian Journal of Zoology, 57(12), 2358-2368. https://doi.org/10.1139/z79-307.

45. Landry, S. M., Koeser, A. K., Kane, B., Hilbert, D. R., McLean, D. C., Andreu, M., \&

46. Staudhammer, C. L. (2021). Urban forest response to Hurricane Irma: The role of landscape characteristics and sociodemographic context. Urban Forestry and Urban Greening, 61. https://doi.org/10.1016/j.ufug.2021.127093.

47. Lepczyk, C. A., \& Warren, P. S. (2012). Urban Bird Ecology and Conservation. Berkeley, CA: Cooper Ornithological Society: University of California Press.

48. Main, M. B., Christman, M. C., Karim, A., \& Hostetler, M. (2011). Species richness and diversity of resident and migratory landbirds in remnant forest patches and residential areas in the Florida Keys, USA. International Journal of Ecology. https://doi.org/10.1155/2011/364213.

49. Marzluff, J. M. (2017). A decadal review of urban ornithology and a prospectus for the future. Ibis, 159, 1-13. https://doi.org/10.1111/ibi.12430.

50. McDonnell, M. J., \& Hahs, A. K. (2015). Adaptation and Adaptedness of Organisms to Urban

51. Environments. Annual Review of Ecology, Evolution, and Systematics, 46(1), 261-280. https://doi.org/10.1146/annurev-ecolsys112414-054258.

52. Merenlender, A. M., Reed, S. E., \& Heise, K. L. (2009). Landscape and Urban Planning Exurban

53. development influences woodland bird composition. Landscape and Urban Planning, 92, 255-263. https://doi.org/10.1016/j.landurbplan.2009.05.004.

54. Møller, A. P. (2009). Successful city dwellers: A comparative study of the ecological

55. characteristics of urban birds in the Western Palearctic. Oecologia, 159(4), 849-858. https://doi.org/10.1007/s00442-008-12598.

56. Oksanen, J. (2011) Multivariate Analysis of Ecological Communities in R: vegan tutorial. Retrieved from http://cc.oulu.fi/ jarioksa/opetus/metodi/vegantutor.pdf. 
57. Petit, D. R., Petit, L. J., Saab, V. a, \& Martin, T. E. (1995). Fixed-Radius Point Counts in Forests: Factors Influencing Effectiveness and Efficiency. Monitoring Bird Populations by Point Counts., 49-56.

58. R Core Team (2020). R: A language and environment for statistical computing. R Foundation for Statistical Computing, Vienna, Austria. URL https://www.R-project.org/

59. Rowan, R., \& Manetz, M. (2006). A Birdwatcher's Guide to Alachua County, Florida (Second Edi).

60. Seewagen, C. L., Slayton, E. J., \& Guglielmo, C. G. (2010). Passerine migrant stopover duration and spatial behaviour at an urban stopover site. Acta Oecologica, 36, 484-492. https://doi.org/10.1007/s10336-010-0605-x.

61. Shanahan, D. F., Miller, C., Possingham, H. P., \& Fuller, R. A. (2011). The influence of patch area and connectivity on avian communities in urban revegetation. Biological Conservation, 144(2), 722-729. https://doi.org/10.1016/j.biocon.2010.10.014.

62. Spurr, E. B. (2012). New zealand garden bird survey - analysis of the first four years. New Zealand Journal of Ecology, 36(3).

63. Sushinsky, J. R., Rhodes, J. R., Possingham, H. P., Gill, T. K., \& Fuller, R. A. (2013). How

64. should we grow cities to minimize their biodiversity impacts? Global Change Biology, 19(2), 401-410. https://doi.org/10.1111/gcb.12055.

65. Sutherland, W., Newton, I., \& Green, R. (2004). Bird ecology and conservation: a handbook of techniques. Oxford: Oxford University Press.

66. Threlfall, C. G., Law, B., \& Banks, P. B. (2012). Influence of landscape structure and human modifications on insect biomass and bat foraging activity in an urban landscape. PLoS ONE, 7(6). https://doi.org/10.1371/journal.pone.0038800.

67. Threlfall, C. G., Ossola, A., Hahs, A. K., \& Williams, N. S. G. (2016). Variation in Vegetation Structure and Composition across Urban Green Space Types. Frontiers in Ecology and Evolution, 4(June), 1-12. https://doi.org/10.3389/fevo.2016.00066.

68. Turrini, T., \& Knop, E. (2015). A landscape ecology approach identifies important drivers of urban biodiversity. Global Change Biology, 21, 1652-1667. https://doi.org/10.1111/gcb.12825.

69. Wilman, H., Belmaker, J., Simpson, J., De La Rosa, C., Rivadeneira, M. M., Jetz, W. (2014).

70. EltonTraits 1 . 0: Species-levelforaging attributes of the world's birds and mammals. Ecology, 95(7), p. 2027.

\section{Figures}

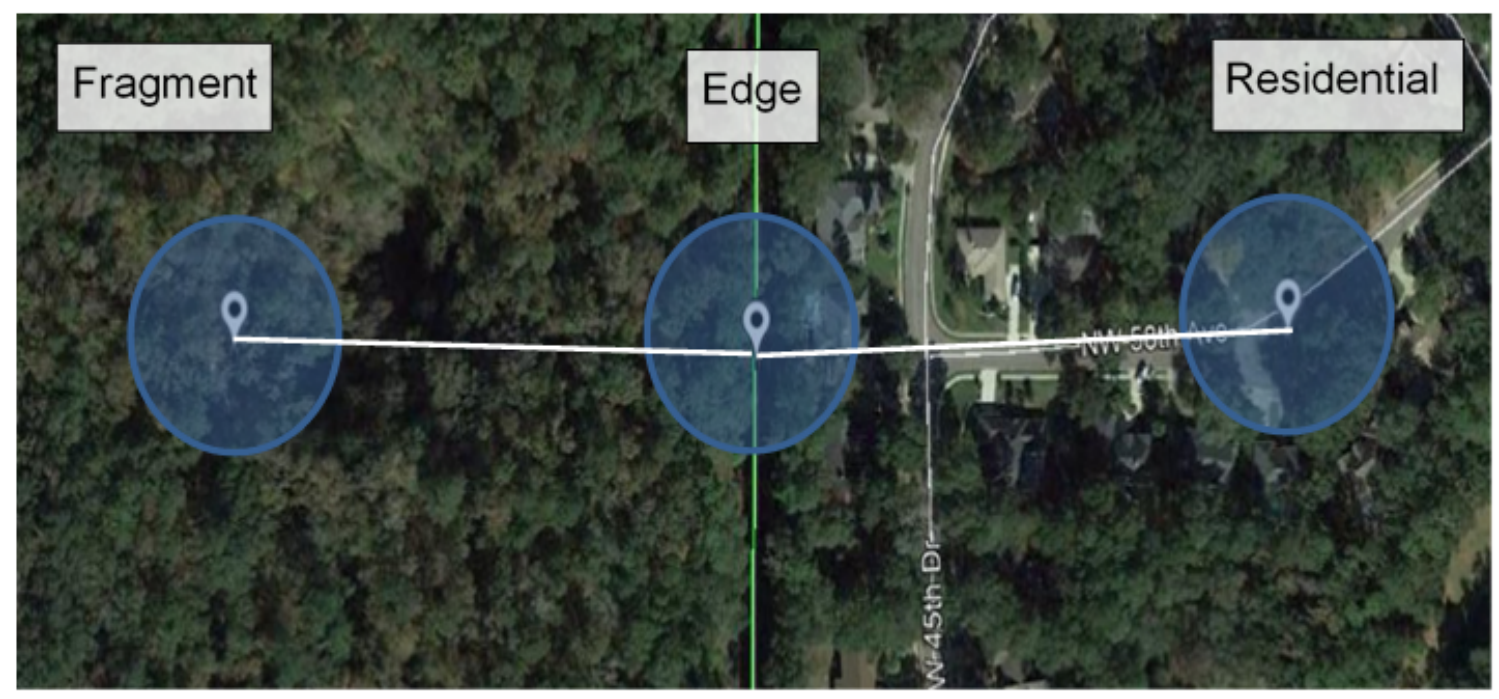

\section{Figure 1}

Diagram of the point counts at each of the 36 survey locations in Gainesville, Florida. Three matched point-counts were done at each location: a) in the forest fragment; b) fragment edge; c) adjacent residential area. The edge-to-fragment point count distance was the equal to the edge-to-residential point count distance. Photo from Google earth imagery. 


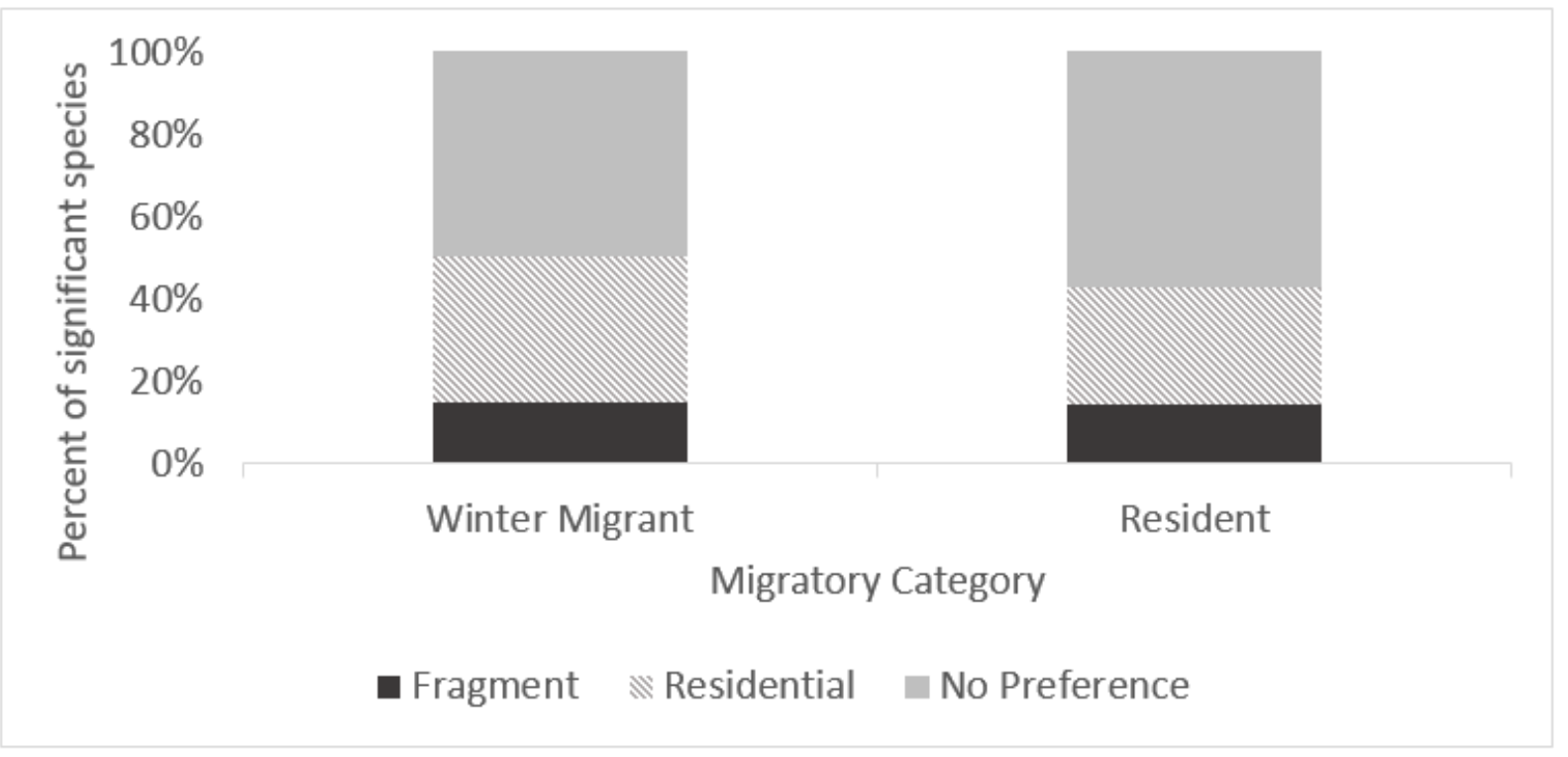

\section{Figure 2}

Bar graph of the migratory guilds representing birds sighted during point count surveys in Gainesville, FL. Bars represent the proportion of species that significantly preferred each habitat type. The black represents fragment preference, the patterned gray represents residential preference, and the solid gray represents no significant preference to a habitat $(n=20$ winter migrants; $n=21$ residents).

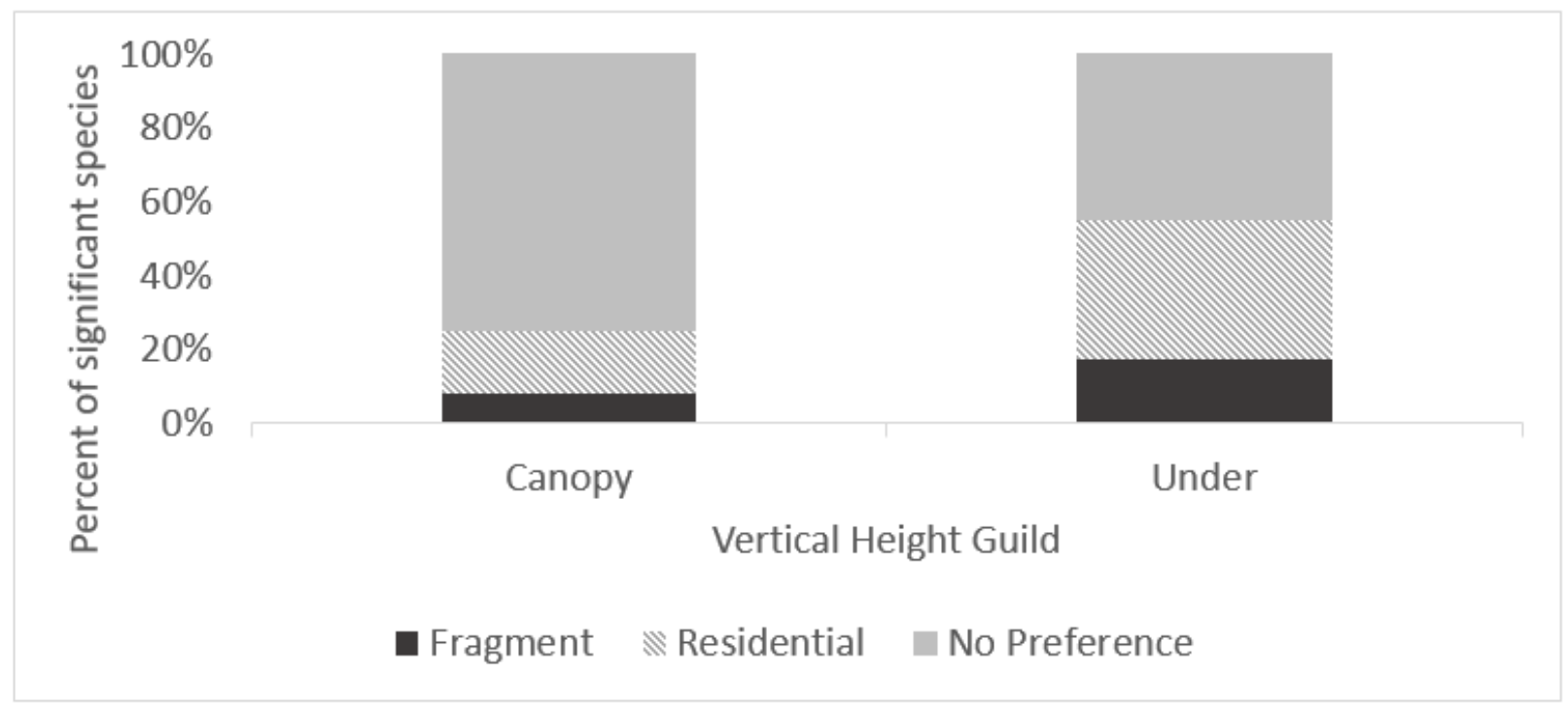

\section{Figure 3}

Bar graph of the vertical strata guilds representing birds sighted during point count surveys in Gainesville, FL. Bars represent the proportion of species that significantly preferred each habitat type. The black represents fragment preference, the patterned gray represents residential preference, and the solid gray represents no significant preference to a habitat ( $n=12$ canopy birds; $n=29$ under canopy birds). 


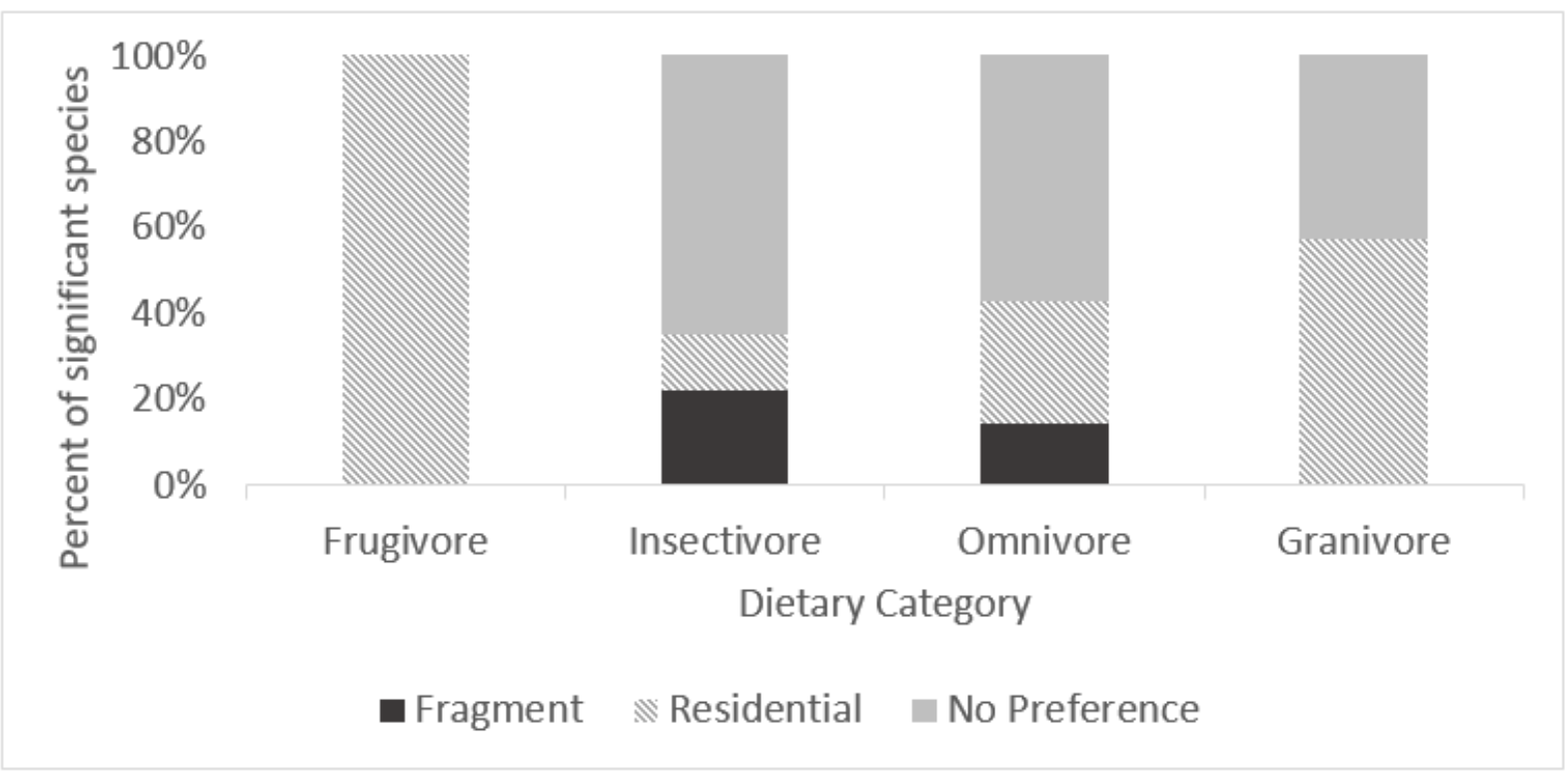

\section{Figure 4}

Bar graph of the dietary guilds representing birds sighted during point count surveys in Gainesville, FL. Bars represent the proportion of species that significantly preferred each habitat type. The black represents fragment preference, the patterned gray represents residential preference, and the solid gray represents no significant preference to a habitat $(n=4$ frugivore birds; $n=23$ insectivore birds; $\mathrm{n}=7$ omnivore birds; $\mathrm{n}=7$ granivore birds). 